\title{
Unveiling the binding mode of adenosine deaminase inhibitors to the active site of the enzyme: implication for rational drug design
}

\author{
Presented by Maria P. Abbracchio
}

Maria Letizia Trincavelli

Published online: 27 January 2013

(C) Springer Science+Business Media Dordrecht 2013

Limongelli V, Marinelli L, Cosconati S, La Motta C, Sartini S, Mugnaini L, Da Settimo F, Novellino E, Parrinello M (2012) Sampling protein motion and solvent effect during ligand binding (2012) PNAS 109:1467-1472.

\section{Summary of the article}

Limongelli and colleagues describe a novel class of potent adenosine deaminase (ADA) inhibitors, developed as drug candidate for the treatment of inflammatory disorders [1]. Since a close correlation has been found between the severity of inflammation and a local increase in both expression and activity of ADA [2], the pharmacological inhibition of this enzyme has recently being regarded as a novel therapeutic approach to counteract inflammation in several pathological conditions. Actually, blockade of the irreversible deamination of adenosine to inosine, normally catalyzed by the enzyme, leads to an increased availability of the biologically active purine at the site of inflammation. Adenosine, in turn, may modulate purinergic responses to these pathological events.

Pursuing their interest in this research field [3-6], and exploiting their synthetic expertise in the synthesis of heterocyclic compounds, authors propose a number of pirazolo[1,5-a]pyrimidin-7-one derivatives bearing suitable alkyl and arylalkyl substituents in the position 4 of the heterocyclic core, which have been shown to inhibit ADA with $K_{i}$ values in the nanomolar range. Although successful in providing novel ADA inhibitors, authors recognize the difficulty of unveiling their mechanism of binding to the

M. L. Trincavelli $(\bowtie)$

Department of Pharmacy, University of Pisa, Pisa, Italy

e-mail: maria.trincavelli@farm.unipi.it active site of the enzyme by means of docking calculations. Actually, both the high flexibility of the protein and the role played by the solvent in accommodating the novel compounds into the ADA site increase the complexity of the interaction between ligands and the target enzyme, thus preventing docking algorithms to clearly describe the binding event at a molecular level.

In the PNAS paper, Limongelli and colleagues brightly overcame this drawback by exploiting well-tempered metadynamics, an emerging technique which allows dealing with protein motion and solvation during ligand binding. With this in hand, they clarified the binding mode of the most active compound, 4-decyl-5-methylpyrazolo[1,5- $a]$ pyrimidin-7(4H)-one, thus reconstructing the free-energy profile of the ligand-enzyme interaction and highlighting the preferred binding mode of the ligand inside the protein.

Besides representing a novel class of ADA inhibitors, the pyrazolopyrimidine here described is an excellent case study of sampling protein motion and solvent effect during ligand binding.

\section{Commentary}

By using a combined experimental and theoretical approach, authors developed a new series of potent non-nucleoside ADA inhibitors and also revealed their mechanism of binding to the enzyme [1]. Pyrazolo[1,5-a]pyrimidine derivatives were generated through rational design, on the basis of pharmacophoric requirements of ADA inhibitors from the literature [2]. Once synthesized by means of conventional synthetic approaches, the novel compounds were tested in vitro for their inhibitory properties on bovine spleen ADA. Finally, their binding modes to the active site of the target enzyme was investigated through a computational strategy 
using a protocol based on metadynamics, which allowed to also clarify the important role played by protein motion and solvent during ligand binding. The originality and the strength of the work resides in the identification, through an innovative and advanced computational technique, of the lowest energy binding mode of the most potent compound of the series, 4-decyl-5-methylpyrazolo[ $1,5-a]$ pyrimidin-7one. These results clarify some of the lead/protein recognition mechanisms at a molecular level and represent a real important opportunity to rationally improve lead compound optimization strategies, protein/ligand interaction, and the pharmacokinetic properties of new ADA inhibitors.

\section{Important insights, caveats, and future directions for research}

Modulation of the level and activity of adenosine represents a very useful therapeutic tool in handling a variety of clinical conditions like ischemia/reperfusion injury, inflammation, and tumor growth [7-9]. Alternatives to adenosine and adenosine receptor agonist/antagonist administration include modulation of adenosine metabolism. Augmentation of nucleoside function by inhibiting its metabolism appears to be a rational therapeutic strategy with distinct advantages: (1) in contrast to specific receptor modulation, the increase of local nucleoside concentrations affects several signal transduction pathways simultaneously and therefore may have the unique potential to modify complex neurochemical networks; (2) by acting at network level, inhibitors of nucleoside metabolism are highly suited to fine-tune, restore, or amplify adenosine physiological functions where needed; (3) inhibitors of nucleoside metabolism have promise for the "soft and smart" therapy of several diseases with the added advantage of reduced systemic side effects.

ADA catalyzes the irreversible deamination of adenosine and 2'-deoxyadenosine to inosine and 2'-deoxyinosine, respectively. ADA exists as cytosolic and extracellular forms and has an important role in regulating intra- and extracellular adenosine concentrations. It is well known that ADA deficiency results in severe combined immunodeficiency by accumulation of lymphotoxic adenosine and 2'-deoxyadenosine [10]. From a pharmacology viewpoint, ADA inhibition has an interest as potential therapy of malignant leukemia and lymphomas. Furthermore, it is appreciated that ADA inhibition has a great potential for anti-inflammatory drugs with few side effects by preventing that adenosine released specifically at inflamed sites is metabolized by extracellular ADA, colocalized at the cell surface with CD26, which is strongly upregulated following $\mathrm{T}$ cell activation and is known as the $\mathrm{T}$ cell activation marker $[2,11]$.

Although a number of ADA inhibitors are known, e.g., pentostatin [12], (+)-EHNA [13], and various other derivatives [4], they have many problems such as poor pharmacokinetics and several toxicities. Pentostatin [14], which is the only ADA inhibitor in clinical use, is only available via intravenous administration, and moreover, use is limited to the treatment of adult patients with hairy cell leukemia. Recent progress has been reported by Terasaka et al., who developed a new generation of ADA inhibitors [15].

The availability of a low number of new ADA inhibitors is due to the complexity in ligand/enzyme interaction at molecular level and to the difficulty to predict a ligand binding mode for the optimization of lead compounds. In particular, the understanding at molecular level of the ligand/ADA interaction has been hampered by the pronounced ability of the active site to accommodate different inhibitors and by the crucial role played by water molecule during ligand binding. Understanding how proteins interact with other molecules (ligands) is crucial when examining enzymatic catalysis, protein signaling, and a variety of other biological processes. It is also the basis for a rational drug design and thus represents an important technological problem. Numerous computational methods have been applied to this problem so as to extract more detailed information. The fastest of these approaches, in terms of speed and computational cost, are based on extensive configurational search of the protein surface (docking), in which the various candidate poses found are scored in accordance with some appropriate function that treats solvation, protein flexibility, and entropic effects in some approximate manner. Nevertheless, for some proteins, including ADA, these protocols failed in predicting ligand-binding recognition site, suggesting the need of using more advanced techniques such as atomistic simulations in explicit water in order to fully include solvent and protein flexibility. Metadynamics has already been used with success to solve difficult docking processes (16) and, by means of this computational approach, authors developed a potential binding mode of the lead compound and also revealed the role of protein motion and solvent during ligand binding. The compound 5-methylpyrazolo[1,5a]pyrimidin-7-one with a decyl chain substitution in position 4 of the heterocycle showed a nanomolar affinity for the enzyme and likely represents a good lead for the synthesis of new potent ADA inhibitors [1].

Authors are to be congratulated on their results, since, by the use of an innovative computational technique, they have been able to shed light on some crucial factors in the interaction of this new class of compounds with the active site of the enzyme. These results thus represent an important starting point for lead optimization strategies in order to develop new ADA inhibitors as potentially active drugs in inflammatory, malignant, and cerebral diseases.

In addition, since in recent years, the medicinal chemistry of ADA inhibitors seemed to have reached a plateau, these findings represent an important breakthrough to revitalize the interest in new drugs targeting adenosine metabolism. 


\section{References}

1. Limongelli V, Marinelli L, Cosconati S, La Motta C, Sartini S, Mugnaini L, Da Settimo F, Novellino E, Parrinello M (2012) Sampling protein motion and solvent effect during ligand binding. PNAS 109:1467-1472

2. Antonioli L, Colucci R, La Motta C, Tuccori M, Awwad O, Da Settimo F, Blandizzi C, Fornai M (2012) Adenosine deaminase in the modulation of immune system and its potential as a novel target for treatment of inflammatory disorders. Curr Drug Targets $13: 842-862$

3. Da Settimo F, Primofiore G, La Motta C, Taliani S, Simorini F, Marini AM, Mugnaini L, Lavecchia A, Novellino E, Tuscano D, Martini C (2005) Novel, highly potent adenosine deaminase inhibitors containing the pyrazolo[3,4-d]pyrimidine ring system. Synthesis, structure-activity relationships, and molecular modeling studies. J Med Chem 48(16):5162-74

4. La Motta C, Sartini S, Mugnaini L, Salerno S, Simorini F, Taliani S, Marini AM, Da Settimo F, Lavecchia A, Novellino E, Antonioli L, Fornai M, Blandizzi C, Del Tacca M (2009) Exploiting the pyrazolo[3,4- $d]$ pyrimidin-4-one ring system as a useful template to obtain potent adenosine deaminase inhibitors. J Med Chem 52(6):1681-92

5. Antonioli L, Fornai M, Colucci R, Awwad O, Ghisu N, Tuccori M, Da Settimo F, La Motta C, Natale G, Duranti E, Virdis A, Blandizzi C (2010) The blockade of adenosine deaminase ameliorates chronic experimental colitis through the recruitment of adenosine A2A and A3 receptors. J Pharmacol Exp Ther 335(2):434-42

6. Antonioli L, Fornai M, Colucci R, Ghisu N, Da Settimo F, Natale G, Kastsiuchenka O, Duranti E, Virdis A, Vassalle C, La Motta C, Mugnaini L, Breschi MC, Blandizzi C, Del Tacca M (2007) Inhibition of adenosine deaminase attenuates inflammation in experimental colitis. J Pharmacol Exp Ther 322(2):435-42

7. Riksen NP, Rongen GA (2012) Targeting adenosine receptors in the development of cardiovascular therapeutics. Expert Rev Clin Pharmacol 5:199-218

8. Lopes LV, Sebastião AM, Ribeiro JA (2011) Adenosine and related drugs in brain diseases: present and future in clinical trials. Curr Top Med Chem 11:1087-1101

9. Gessi S, Merighi S, Fazzi D, Stefanelli A, Varani K, Borea PA (2011) Adenosine receptor targeting in health and disease. Expert Opin Investig Drugs 20:1591-1609
10. Resta R, Thompson LF (1997) SCID: the role of adenosine deaminase deficiency. Immunol Today 18:371-374

11. Kameoka J, Tanaka T, Nojima Y, Schlossman SF, Morimoto C (1993) Direct association of adenosine deaminase with $\mathrm{T}$ cell activation antigen, CD26. Science 261:466-469

12. Agerwal RP, Spector T, Parks RE (1977) Tight-binding inhibitors. IV. Inhibition of adenosine deaminase by various inhibitors. Biochem Pharmacol 26:359-367

13. Bessodes M, Bastian G, Abushanab E, Panzica RP, Berman SF, Marcaccio EJ, Chen SF, Stoeckler JD, Parks RJ (1982) Effect of chirality in erythro-9-(2-hydroxy-3-nonyl) adenine (EHNA) on adenosine deaminase inhibition. Biochem Pharmacol 31:879-882

14. Flinn IW, Kopecky KJ, Foucar MK, Head D, Bennett JM, Hutchison R, Corbett W, Cassileth P, Habermann T, Golomb H, Rai K, Eisenhauer E, Appelbaum F, Cheson B, Grever MR (2000) Longterm follow-up of remission duration, mortality, and second malignancies in hairy cell leukemia patients treated with pentostatin. Blood 96:2981-2986

15. Terasaka T, Tsuji K, Kato T, Nakanishi I, Kinoshita T, Kato Y, Kuno M, Inoue T, Tanaka K, Nakamura K (2005) Rational design of non-nucleoside, potent, and orally bioavailable adenosine deaminase inhibitors: predicting enzyme conformational change and metabolism. J Med Chem 48:4750-4753

16. Masetti M, Cavalli A, Recanatini M, Gervasio FL (2009) Exploring complex protein-ligand recognition mechanisms with coarse metadynamics. J Phys Chem B 113:4807-4816

\section{About the author}

Maria Letizia Trincavelli, Ph.D. is a tenure Assistant Professor in Biochemistry at the Department of Pharmacy, University of Pisa, Italy. She has been working in the purine research field for almost 15 years and her current research interests focus on P1 and P2 purinergic receptors, their transduction systems and regulatory processes (desensitization-internalization) both under physiological and pathological conditions, including inflammation and brain diseases. In recent years she has crucially contributed to the cloning, "deorphanization," and pathophysiological characterization of the P2Y-like receptor GPR17 and she is investigating the role of receptor desensitization in oligodendrocyte differentiation in demyelinating diseases. 